\title{
Generating Realistic Dynamic Prices and Services for the Smart Grid
}

\author{
Giuliano Andrea Pagani and Marco Aiello \\ Distributed Systems Group \\ Johann Bernoulli Institute for Mathematics and Computer Science \\ University of Groningen \\ Groningen, The Netherlands \\ email: \{g.a.pagani,m.aiello\}@rug.nl \\ http://www.cs.rug.nl/ds/
}

JBI technical report: JBI 2013-12-1

January 1, 2013

\begin{abstract}
The Smart Grid promises to change the way end-users manage their energy needs, enabling the accommodation of renewable sources and providing dynamic and flexible tariffs. These will vary through the day to reflect the actual availability of energy and the congestion conditions of the Power Grid. We present a vision of the services needed for the end-user to enable the Smart Grid in a Smart Home and we illustrate an implementation simulating the behavior and services offered. The simulation is realistic in its offering of dynamic prices and services gathering the data from actual sources. The aim is to offer a testing platform and to show the potential benefits of dynamic pricing and energy self production, the impact on the user and the possibility to easily realize testbeds and pilot projects. We have tested the proposed Smart Grid services and Dynamic Pricing solution in a smart building experiment in an existing living lab.
\end{abstract}

\section{Introduction}

The power industry is at the verge of major change similar to the one that invested the society at the beginning of the electricity era. The availability of renewable energy sources, the digitalization of the infrastructure and a set of unbundling regulations are changing the energy distribution and generation landscape. Recently, the term Smart Grid is gaining momentum. Though it does not yet a final and unique definition [1]. The new (Smart) Grid will be able to diagnose itself and take appropriate action in case of faults, therefore it will become more resistant to willing and unwilling attacks and disasters. Another key feature will be the ability of the Grid to accommodate more renewable-based 
sources of power. This last aspect is particularly challenging since renewable sources introduce substantial variability and unpredictability in the energy system, which is particularly challenging and it puts the system under stress. Last but not least, the end user will have an electrical system that has the same or even improved power quality and reliability meeting his needs and expectations. In addition, the end users will benefit by the possibility to interact and respond to electricity price signals in a multi-provider and multi-tariffs energy market, thus potentially save on his energy bill. Such vision is shared by many key players as, for instance, the U.S. Department of Energy (cf. [2] and [3]).

By transitioning to the Smart Grid, the power industry is becoming even more a system of systems. The already heterogeneous power system world including generation plants, control equipment, supervisory control and data acquisition (SCADA), transmission and distribution lines and meters is being split in even more subsystems that belong more to the category of information and communication technology (ICT). In addition, more actors can now plane in any given role as the road to unbundling of the sector has been taken by many countries. ICT will be the key to enable distributed control of small scale distributed energy resources, to enable the remote control of digital meters, to interact with the users and their appliances through variable tariffs.

The large interest in Smart Grids and the somewhat shared general vision, though is not yet paired with any large scale deployment. Researchers currently face the problem of testing ideas and solutions against an envisioned but not available infrastructure. For this reason, we have designed and implemented a system that, on the basis of actual market and environment data, can be used for Smart Grid pilot projects involving users, to realize Smart Grid compliant devices and to guide Smart Grid products research and development (e.g., intelligent appliances). In fact, the houses, offices and industries of tomorrow will benefit by adapting their energy consumption patterns to the price and availability of energy. In particular, considerable advantages from an economical point of view will come from the possibility of having a dynamic price structure (also known as real-time pricing), and from an environmental one by the use of decentralized small-scale generation facilities based on renewables.

\subsection{Demand-Response}

Demand-Response is the mechanism to couple end user energy request with Grid's energy supply in real-time. Such definition is closely related with Dynamic Pricing and variable tariff. Even though Dynamic Pricing is not yet available to the end user, the transition towards more prices per day and switching among energy providers rapidly is underway. In many countries, the energy business panorama has moved from a single provider/single tariff system, to models with competing providers. Generally, two, or anyway a very limited number of pre-fixed tariffs are used; prices are fixed by long term contracts, usually in the term of months. E.g., an energy provider can offer distinct tariffs for daytime and night-time or weekdays and week-end, the so called peak and off-peak tariffs. In this way, the energy providers want to incentivize the users to balance their demand with the supply. Such need is due to the fact that the costs of increasing the energy supply do not increase linearly with the demand, but they are rather a convex function which is composed by linear intervals with increasing slope as the energy used increases [4]. In the future, the situation will 
be even more delicate with the adoption of renewable sources entailing supply irregularity and uncertainty [5].

Renewable energy sources are and will be more and more present not only at the medium-large scale on the Grid, but also at the level of the single building. Energy solutions based on solar panels, small wind turbines and combined heatpower generators from gas and bio-mass will be more and more common [6]. The so-called Smart Home of tomorrow will have to be aware both of the energy generated locally and the prices of energy on the market in order to choose the proper policies to adopt: either use the energy produced for local needs or feed the energy into the Power Grid and receive a payment for it. Therefore, the intelligent elements inside the house have to be able to know the energy produced on-site in order to eventually adapt their operations. As with every commodity, the goal at household level is that of saving money on the energy bill, while keeping an adequate level of well-being, comfort and satisfaction for its inhabitants. This monetary goal translates into three practical objectives: reducing the overall consumption of energy, adding attractive forms of local energy generation, and buying energy at the lowest possible price.

To answer these challenging objectives, we present a simple example on monitoring, forecasting and pricing of energy produced through renewables and we show how to create a Dynamic Pricing mechanism based on real data by using current Internet and web technologies. The realized module is a valuable tool for simulating futuristic interactions with the coming Smart Grid. The tool we describe in this paper can be used in today's research and development of products and solutions for the future Smart Grid. In addition, it can be used to test the feasibility of Smart Grid-enabled home automation solution and also to make simulations of production of renewable sources to be used on-site. Another functionality of our tool is to provide an economic evaluation of electricity generated by renewable sources compared to traditional energy markets. The proposed solution has been implemented in an actual test bed in an office environment where electrical appliances are scheduled based on user preferences and dynamic prices [7].

\subsection{Smart metering}

The Smart Meter is the energy entry point to smartness for the household. Though, Smart Meters in some countries have been already widely deployed (e.g., Italy and Sweden), their current functionality is essentially the one related to Automated Metering Reading (AMR). It is a bidirectional flow of communication between the meter and the energy provider. This flow of information enables the utility to read meter data for the energy consumed (or produced and fed into the Grid) by the customer, to remotely attach new customer, or detach moved or non-paying customers. Figure 1 provides a representation of this traditional landscape of Smart Metering where the Smart Meter is essentially a "Dumb Meter" enriched only by communication capabilities.

In a Smart Grid enabled environment the Smart Meter promises to be an important element. In addition to the functionalities available in the current Smart Meter landscape, we envision a Smart Meter capable of interacting on a multi-provider energy market characterized by Dynamic Pricing. The meter can set the tariffs accordingly and can interact with household appliances and with the end-user that defines his desired spending caps and time of use for appli- 


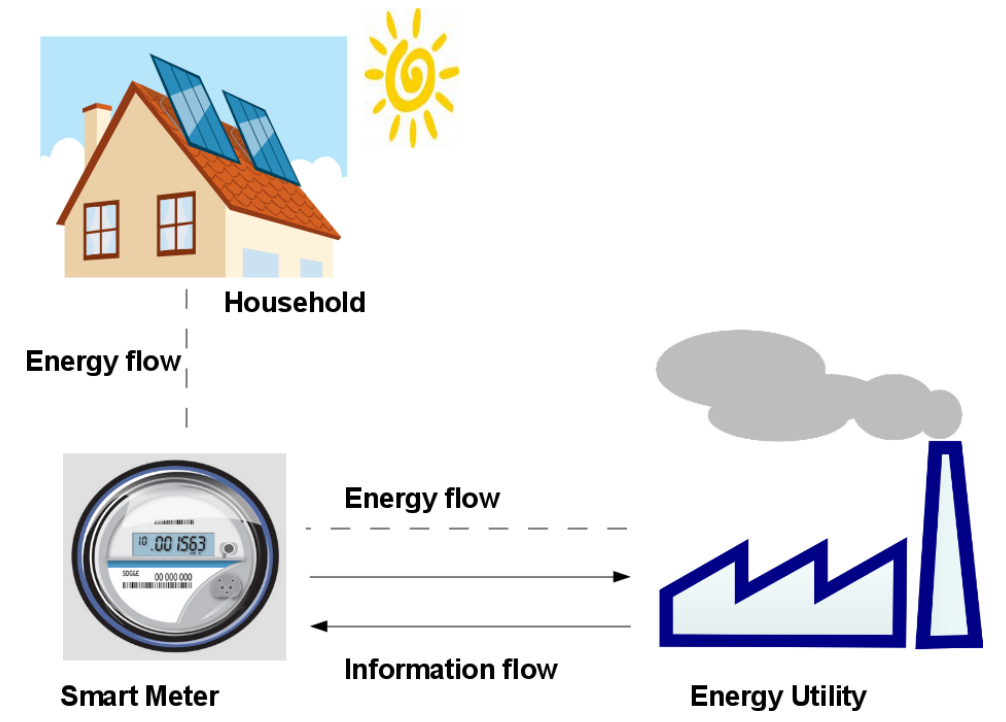

Figure 1: Current Smart Meter usage.

ances (e.g., the dishwasher has to be completed before dinner time). Moreover, the Smart Meter can access the Internet to gather information about environmental conditions that might influence local energy production (e.g., rooftop solar panels) and provide the end-user with an energy production forecast and a possible schedule for appliances, see Figure 2. In other words, it will become the front end for home energy management.

It is therefore essential to be ready and test the Smart Grid functionalities affecting the household. Such evaluation will be in terms of savings through Dynamic Pricing, user experience, tests on the scheduling of appliances and Smart Meter software that interacts with the Smart Grid services. A platform for simulations with realistic data is the first essential step to enable the development of new products and services that can benefit from the future Smart Grid. Users are those who will take advantage of products (e.g., smart appliances) and services (e.g., cost saving scheduler software application for Dynamic Pricing) optimized for the Smart Grid. The simulator we propose here is an essential element that is needed to properly develop and test such products and solutions.

\subsection{Paper Organization}

The paper is organized as follows. Section 2 describes the the current state of the art on the Smart Grid services topic. A comparison of the ideal and actual solutions for realizing services for Demand-Response in the Smart Grid are described in Section 3, while an example of the implementation follows in Section 4 . Section 5 provides the results and output description of the realized Smart Grid services. Section 6 concludes the paper with a discussion of the pros and cons of the approach. 


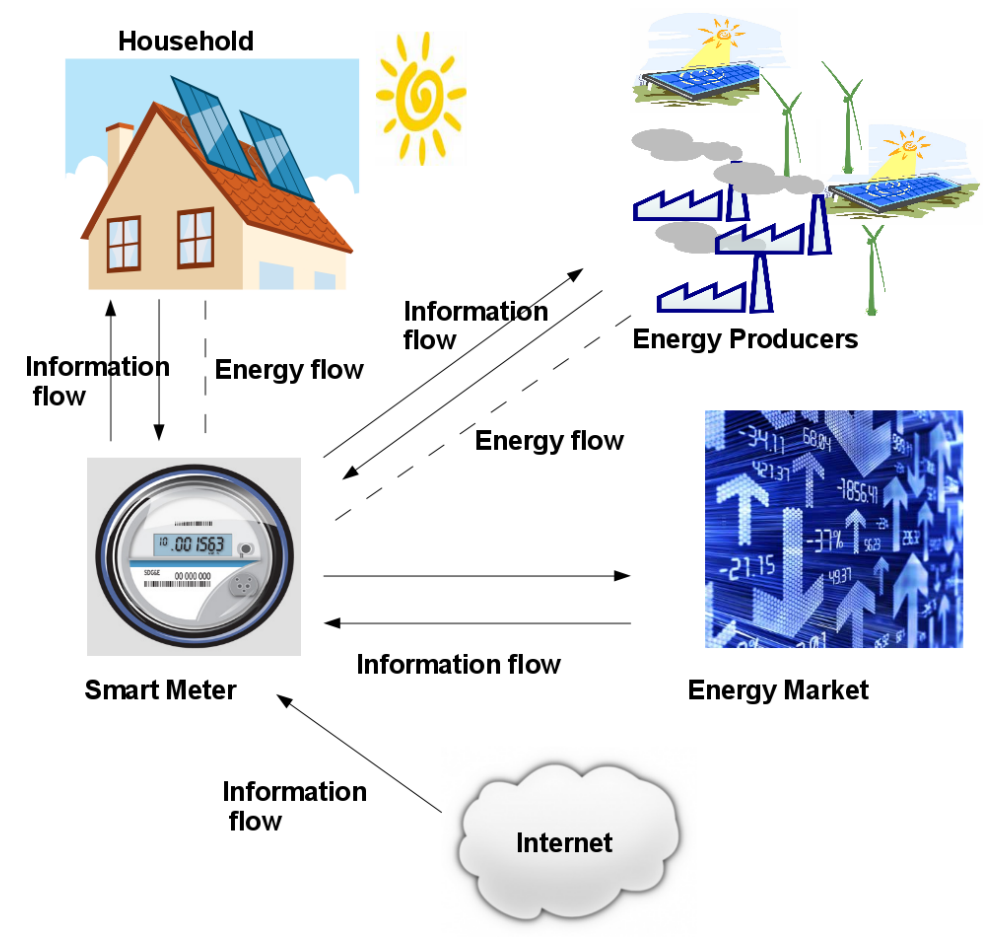

Figure 2: Envisioned Smart Meter usage.

\section{Related Work}

The Smart Grid has grown in popularity in the past few years and several approaches and architectures have been proposed for the information services related to the Smart Grid for the Smart Home. Most of the works are architecture proposal with interfaces to services that are implemented abstractly or based on historical energy data, thus, missing to model the real-time dynamics of the system.

In [8], the authors simulate the effects of aggregate households appliances and electric vehicles to act as balancing devices for solar installations. They show the feasibility of reducing the amount of spinning reserves and the benefits in terms of reduced $\mathrm{CO}_{2}$ emissions, considering several types of solar plants and scenarios of electric vehicles penetration. The work is entirely a simulation effort with no real services involved. Another simulation tool for Demand-Response is proposed in [9]. The goal is to analyze at a very detailed level (bus voltages, power flows, etc.) a control strategy to accommodate the wind power of a turbine and its fluctuations (0.5 MW of power) in a small/medium scale community (650 buildings). The model shows that by applying a control strategy to the heath pumps in the buildings one can provide a considerable smoothing in the variations of power in the slack bus of the simulated IEEE 13 bus system. The presented system is a complex MATLAB simulation environment and deals with the electrical problem and not with the ICT or Internet-based services to enable the Demand-Response in practice. The importance of services in the future Smart Grid is emphasized by Karnouskos [10] who considers the services 
as the only way to let the various actors in the Smart Grid panorama interact with each other. It is more a vision/position paper than an analysis and the work does not propose specific services, technologies or implementations to be used. The work of Strobbe et al. provides an architecture for the future Smart Grid that is built on a JAVA-based standard protocols. The services provided are mainly proposed to engage and raise the awareness of the user to energy conservation and peak reduction by having information of prices. The architecture presents interesting aspects such as the ease of extension and the energy price individuation via the Belgian power exchange trading market. However, there is no mention of integration of renewable sources and energy forecast in the architecture, these are essential building blocks of the future Smart Grid. In addition, the work does not consider any automation process towards appliances, but just the user interaction with an home display that provides consumption and tariff information. Another example of an architecture based on services is given in [11]. Although the amount of services proposed for the future Smart Grid is considerable (energy monitoring, prediction, management, optimization, billing, and brokerage) and well described also in the technical details of the architecture (e.g., Representational State Transfer), there is little to no description of the implementation of such services. There are no details of data sources in order to implement such architecture in practice. Another architecture for the future Grid is proposed in [12]. The authors consider a service architecture that can easily plug-in new services. The architecture can host smart applications that are able to interact and control devices, and act as a contact point with the services provided by service-providers related to energy (e.g., real-time pricing, remote device control). The simulation of the Smart Grid is obtained through the OMNeT ++ framework where the information about topology, user consumption and scenario parameters are provided. Such architecture is valuable and enables a good simulation in the large of the Smart Grid. However, it misses the interaction with real information and data that are the key to provide more concrete and valuable results to the mere simulation exercise. The model presented in [13] focuses on simulating a multi agent system where each agent is an energy consumer or producer. The simulation is based on historical demand patterns, while in the energy generation patterns, context information for solar and wind production are taken into account through the interaction with web services. Although the approach is interesting and uses real data, it lacks an important ingredient of the Smart Grid that is Dynamic Pricing. Moreover, there is no mention of energy forecast which essential in the schedule operations of home appliances, and the time granularity considered in the work is quite long (three hours) compared to the dynamics of the Smart Grid. A different approach in providing the functionalities of the Smart Grid in Smart Home is given by the development of hardware components for home energy management. These devices learn users' patterns of electricity usage, monitor the external condition of the Power Grid and enable to control devices [14]. However, in the work there is no evidence of sources of information for environmental conditions, tariff differentiation is not in the picture and the approach focuses on the hardware rather than the software/service aspects. A residential energy management system is described in [15]. In such paper, the DemandResponse is well described, there are several devices and scenarios taken into account (dishwasher, dryer, electric vehicle, refrigerator, etc.), and a return on investment analysis is performed. Though, there are some limitations. First, 
it is only a simulation and there is no interaction with real equipment or real services providing consumption information; second, there is no tariff differentiation and the Demand-Response is only available in a time window during the day; third, distributed energy generation through renewables is not mentioned. Demand-Response modeling is the focus of [16] where consumer's attitude to modify his habits is investigated through price elasticity in the case of a microgrid. Although modeling and running simulation to evaluate the benefits of dynamic pricing is essential, the work lacks in actual price data (the authors use prices of just one day). In addition, there is no mention of the usage of selfproduced energy and that, actually, could change the attitude towards price elasticity (buying a small quantity of additional energy at a high price while most of it is self-produced). Web services are the key components to interact in a Smart Grid-enabled home as suggested in [17] and [18]. These works, however, are mostly an exercise in web service interaction and simulation of web services communication (representing appliances and sensors) rather than a real set of services or components that can be applied in a Smart Grid test solution. The data related to energy consumed in the home environment is the U.S. average, the energy extractable by solar panels and wind turbine are static and with no variation according to environmental condition, moreover only peak and offpeak tariffs are considered. Such approach of simulating the Smart Grid is quite simple and lacks in the dynamism of the Smart Grid components (i.e., energy prices, renewable energy production) which are all the crucial aspects of the Smart Grid. Considering the broader aspect of the whole interactions between the many actors of the Smart Grid, service-oriented architectures have been proposed in several works to be the good candidates to solve interoperability issues $[19,20,21]$.

In summary, we note that effort has been spent in the simulation of Smart Grid aspects; while there is a gap in the investigation of how the future Smart Grid services should be and how they should work. Even more problematic is that the data and information used to simulate the Smart Grid (e.g., Dynamic Pricing, usage of renewable energy) are usually based on limited historical data, often averaged out, rather than based on real-time actual data.

\begin{tabular}{|c|c|c|c|}
\hline Category & Vision & $\begin{array}{l}\text { Ongoing standardization ef- } \\
\text { fort }\end{array}$ & Nowadays realization \\
\hline Energy price & $\begin{array}{l}\text { Third party web service provid- } \\
\text { ing prices from several energy } \\
\text { providers }\end{array}$ & $\begin{array}{l}\text { OpenADR, ZigBee Smart En- } \\
\text { ergy Profile 2.0, OASIS EMIX, } \\
\text { IEC-62325, direct interaction with } \\
\text { Smart Meter }\end{array}$ & $\begin{array}{l}\text { Ad hoc data retrieval in real wholesale } \\
\text { energy market }\end{array}$ \\
\hline $\begin{array}{l}\text { Energy produc- } \\
\text { tion/ consump- } \\
\text { tion }\end{array}$ & $\begin{array}{l}\text { Web service in the control unit of } \\
\text { the small-scale production plant }\end{array}$ & $\begin{array}{l}\text { OpenADE, ZigBee Smart Energy } \\
\text { Profile 2.0, IEEE 1547.3, direct in- } \\
\text { teraction with Smart Meter }\end{array}$ & $\begin{array}{l}\text { Ad hoc interaction with produc- } \\
\text { ing/consuming device control and } \\
\text { monitoring equipment, interaction } \\
\text { with Smart Meter of a specific } \\
\text { standard/technology }\end{array}$ \\
\hline $\begin{array}{l}\text { Environmental } \\
\text { conditions }\end{array}$ & $\begin{array}{l}\text { Dedicated web service from mete- } \\
\text { orological companies specialized in } \\
\text { energy with very localized forecast- } \\
\text { ing }\end{array}$ & $\begin{array}{l}\text { Outcome of NIST B2G DEWG, } \\
\text { addition to existing weather stan- } \\
\text { dards e.g., Weather Information } \\
\text { Exchange Model (WXXM) }\end{array}$ & $\begin{array}{l}\text { Interaction with existing weather web } \\
\text { services (e.g., Yahoo!, NOAA, The } \\
\text { Weather Channel) }\end{array}$ \\
\hline
\end{tabular}

Table 1: Smart Grid services: vision, ongoing standardization processes, and nowadays possible realization.

In Sections 3 and 4, we show how our simulator of the Smart Grid fills 
the gaps highlighted in the state of the art just presented. Our Smart Grid simulator provides dynamically changing information and uses realistic up-todate information from web-based sources. It also considers the key components to test Smart Grid-enabled scenarios such as Dynamic Pricing, forecast and production of energy by renewable small-scale sources.

\section{Smart Grid services: vision and current real- ization}

The Smart Grid vision is far from being implemented, at most there are local and small scale testbeds (e.g., Hoogkerk's PowerMatching city Smart Grid project [22]) where some features are implemented. In addition, business analysis and business cases around the Smart Grid are still under investigation [23, 24]; so is the standardization process. For a comprehensive overview we refer to our previous work [19]. The Smart Grid is still rich of uncertainties, however some functionalities are assumed to be its pillar: Demand-Response through Dynamic Pricing, local energy generation through renewables, and energy forecast to help schedule smart appliances when more favorable. In such scenario, our aim is to design and realize a Smart Grid Simulation Engine based on available web resources. We have developed ad-hoc interfaces to existing services on the Web (e.g., energy tariffs, meteorological forecast for renewable energy) in order to realize the Smart Grid features essential for a Smart Meter/Smart Home interaction. In particular, information regarding energy prices, energy generated, and environmental conditions is accessed through web sites or web services. For each Smart Grid functionality such as energy price, energy production metering and meteorological conditions, we describe the solution we envision to be available in the future, but we also describe what can be realized today with the currently available information. A recurrent aspect for the envisioned ideal solution is the use of Service-Oriented Architectures (SOA). SOAs are the most recognized framework to implement large scalable and dynamic information systems, such as the Smart Grid [19]. A typical implementation of SOA is through web services. Web services are a common way of realizing interoperability between heterogeneous systems over a network by exploiting the publish-subscribe-bind pattern [25]. An incarnation of web services is through protocols like the Hypertext Transfer Protocol (HTTP), the Simple Object Access Protocol (SOAP), and through standardized service descriptions interfaces such as the Web Services Description Language (WSDL). A summary of the vision for the Smart Grid services, the standardization process involved, and the nowadays possible implementation is shown in Table 1.

\subsection{Interface towards energy price service}

The availability of updated prices and price forecast within few hours or within few days are essential to implement the Smart Grid Demand-Response functionality. 


\subsubsection{Vision}

The envisioned solution to implement the price mechanism would be a web service provided by a third party service for the Smart Grid that administers the energy tariffs for the providers that the Smart Home is allowed (or wants) to supply from. The Smart Home subscribes to this service indicating for which providers the tariffs are required. Either in a pull or push method the Smart Home obtains the forecast tariffs for different time granularity. The granularity can be real-time for balancing purposes or hourly day-ahead forecast for mid/long term appliances schedule. The service, in addition, signals through a push message a changing tariff compared to the previous forecast so that energy intensive operations already scheduled in the house can be adapted on-the-fly to the changing price conditions. In this ideal situation all the interactions and information exchange happen automatically thorough the World Wide Web and the Smart Meter. The OASIS Energy Market Information Exchange (eMIX), still under development at the moment, might be the protocol used to exchange information about energy prices.

\subsubsection{Realization today}

The solution proposed above is ideal and not implemented at the moment. However, it is possible to have a similar behavior by interacting with wholesale energy markets that already implement price differentiation in the energy trading process. In particular, the prices generated on the wholesale market vary in accordance with the congestion and energy availability on the Power Grid infrastructure. This is realized to keep the balance between demand and supply, in other words the Demand-Response mechanism. To have variable energy tariffs it is possible, for instance, to use prices coming from the PJM Interconnection ${ }^{1}$ which is a regional transmission organization (RTO) that coordinates the movement of wholesale electricity in more than 13 states of Eastern U.S.A., or from another organization such as ISO New England (ISO-NE) ${ }^{2}$ which provides the energy market facilities for the New England region of the U.S.A. For both markets the data that can be extracted are the real-time prices and the Day-Ahead Energy Market locational marginal pricing (LMP) which are respectively the live minute-by-minute prices and the prices of energy negotiated in the wholesale market for the following day by energy companies at specific locations where energy is delivered or received. For the day-ahead market, data contain the energy price for each unit (dollars per Megawatt-hour) for each hour of the day (for the next day) at the locations of delivery. Each location can have in principle a different price from any other one. These prices can be considered for the Smart Home as the prices of different energy providers. Real-time prices can be obtained by repetitively interrogating the PJM or ISONE web site where prices for the various hubs are continuously updated; while the day-ahead data can be automatically obtained through PJM or ISO-NE web site and they are available each day for the following day (day-ahead) as a Comma Separated Value (CSV) files. Little ad hoc adaptations are required to automatize the process of file download and cleaning of the unnecessary fields, before they can be used as the input of a systems that provides Dynamic Pricing

\footnotetext{
${ }^{1}$ http://www.pjm.com/

2 http://www.iso-ne.com/
} 
for Demand-Response purposes. A feasible solution today is to use Day-Ahead prices to forecast the schedule for smart appliances for the following day, and then correct the scheduling in real-time if considerable difference in prices arise in the real-time market.

\subsection{Interface towards energy producing equipment service}

Another essential feature for a Smart Home is the possibility of generating energy through small-scale renewable plants (e.g., photovoltaic panels, small wind-turbine)

\subsubsection{Vision}

The envisioned solution to implement information retrieval of the amount of energy and power produced by a small-scale energy plant would be a web service provided by the control unit of the plant. The Smart Home, directly or through the Smart Meter, could then in an automated way ask the information about the available instantaneous power and the amount of energy generated. Information about the generated power could be easily automatically reported via web service interaction to the utility company with whom the sell contract is signed if no Advanced Metering Infrastructure system is already implemented.

\subsubsection{Realization today}

Usually, every modern small-scale plant has an electronic controller that is able to show information about power and energy produced together with essential technical and environmental parameters of the plant (e.g., for a photovoltaic plant: air temperature, panel temperature, and solar irradiance). This information is usually published on the Internet (or LAN) through the web server embedded in the controller of the plant so that the owner of the plant can monitor the performance. This information can usually be directly accessed or downloaded in CSV format so that with little ad hoc manipulations they can be used as input for an application. An example is available at the U.S. School Power Naturally data portal. ${ }^{3}$ Through this portal it is possible to access solar plant information and data. Another example of a web server is available at http://pagani.dyndns .org/html/en/onlineOverWr.html, it provides real-time and historical information from a solar plant about the power and energy produced. While a comprehensive small-scale power generating unit composed by a solar, wind turbine and small-scale combined heat and power turbine plant is available at Grand Valley State University. ${ }^{4}$

\subsection{Interface towards environmental service}

External environmental and weather parameters are essential to know how external conditions may influence both energy consumption inside the Smart Home and the renewable-based generating equipment. The Smart Home has then to be able to access current and forecasted environmental conditions.

\footnotetext{
${ }^{3}$ http://sunviewer.net/portals/NYSERDA/

${ }^{4}$ http://datamonitoring.marec.gvsu.edu/
} 


\subsubsection{Vision}

The envisioned solution to obtain environmental parameters would be a dedicated web service provided by a third-party specialized in environmental conditions forecast for the Smart Grid with precise geolocalization. In addition to traditional weather information (e.g., weather conditions, temperature, pressure), other information such as solar irradiance, wind speed, humidity, cloud coverage, air density, external light conditions and others are significant to estimate energy consumption of buildings and to forecast energy production by small-scale energy equipment. This information should be extremely localized and dedicated to the specific location of a Smart Home. The envisioned service requires that the Smart Home subscribes to the service by providing exact location (longitude and latitude coordinates) and orientation towards the sun. Once subscribed, the service should be able to provide the environmental parameters mentioned above. The service could be implemented in a push and pull manner so that updates on the conditions and forecasts can be sent to the Smart Home regularly, or it can request for an update when needed.

\subsubsection{Realization today}

On the World Wide Web there are several weather services, with advanced pieces of functionality that can be accessed in the form of web services. A primary example, considering the levels of detail and abundance of weather-related parameters (current weather, 7 day forecast and many parameters such as max and min temperatures, wind speed, wind direction, wind gust, percentage of sky cover and more), is provided by the National Weather Service realized by the National Oceanic and Atmosphere Administration (NOAA) ${ }^{5}$ in the U.S.A. From a technical point of view the service provides an interaction through SOAP requests. Other services that use a web service approach are provided by commercial services such as Yahoo!. ${ }^{6}$ This weather-related information seems the closest to the ideal solution for this kind of service required by the Smart Home in his interaction with the Smart Grid.

\section{Implementation}

Proof-of-concepts implementations of the Smart Grid, simulations of customer behavior or small scale pilot projects have been reported being underway [26, 27, 28], though no actual large implementation exists.

To be able to perform research and development related to Smart Grid, we have developed Smart Grid services considering the case of a Smart Home located in New York, U.S.A. with the ability of generating energy with smallscale production units through a photovoltaic (PV) installation of $2.4 \mathrm{~kW}$ of power realized using an AstroPower AP-100 PV module ${ }^{7}$, and a small-scale wind turbine namely the Proven 2.5 wind turbine. ${ }^{8}$

In our simple implementation scenario for Smart Grid interaction we decided to use data and services coming from real markets, real energy installations, and

\footnotetext{
${ }^{5}$ http://www.noaa.gov/

${ }^{6}$ http://developer.yahoo.com/weather/

${ }^{7}$ http://atlantasolar.com/pdf/Astropower/ap-100.pdf

${ }^{8}$ http://www.windandsun.co.uk/Wind/wind_proven.htm
} 
real weather information. Such choice enables us to realize a realistic simulation of Dynamic Pricing functionality, energy production from small-scale renewable sources and energy forecast. A representation through a block diagram of the components involved and implemented in software using JAVA programming language is shown in Figure 3. The central component is the Smart Grid Simulation Engine which is responsible for contacting the information providers. Its role is to establish external connections, gather the data and convert them to the format required by the internal sub-component that has then specific tasks to further process those data.

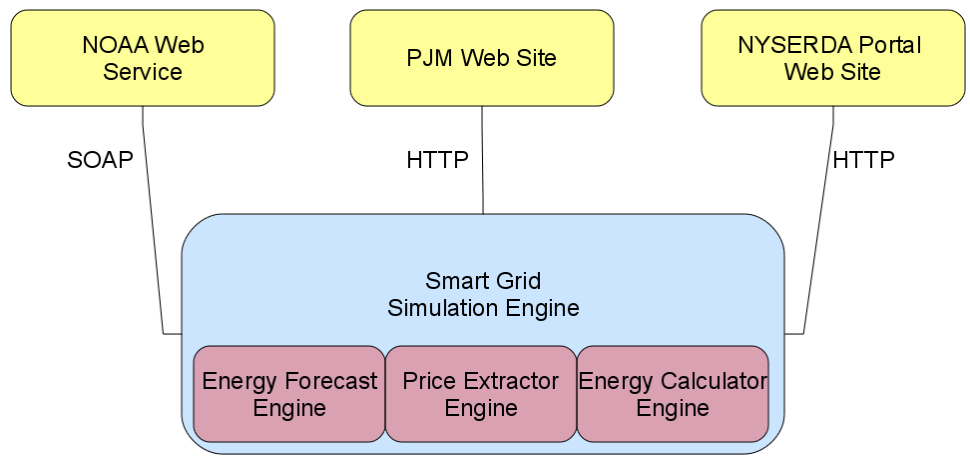

Figure 3: Smart Grid simulation engine and external services.

To simulate the variable energy tariffs, we use the energy prices coming from the PJM Interconnection and the data extracted is the Day-Ahead Energy Market LMP. The Smart Grid Simulation Engine makes an HTTP request to the PJM web site where Day-Ahead LMP prices are stored as CSV files. The data contain the energy price for each unit (dollars per Megawatt-hour) for each hour of the day (agreed for the following day) at 20 locations of delivery. We consider that the energy supply of a home could be fulfilled not just by one provider at a time, but also by the composition of several providers that might contribute to satisfy the power required by a house (e.g., provider A is able to give $1 \mathrm{~kW}$ of power at tariff $x$ and provider $\mathrm{B}$ is able to give $2 \mathrm{~kW}$ of power at tariff $x+\Delta$ for a certain time period).

To account for the energy produced in the Smart Home, we access a real PV installation in New York at Dalton School in Manhattan ${ }^{9}$ that has the same PV array hypothesized in our Smart Home and whose real-time data can be accessed through the U.S. School Power Naturally data portal. The Smart Grid Simulation Engine connects to the web site ${ }^{10}$ providing the performance of the solar installation on top of the school, and extracts the CSV file with realtime information about the power generated by the PV array. Data is sampled every 15 minutes. Information about relevant environmental parameters such as solar irradiance, ambient temperature and wind speed is also available. For our purpose, we assume a simplified situation: the data about the power is available every hour and constant.

To simulate the power supplied by the wind turbine in the Smart Home, we use the data gathered by the anemometer at Dalton School. The CSV

\footnotetext{
${ }^{9}$ http://www.dalton.org/

${ }^{10}$ http://sunviewer. net/portals/NYSERDA/siteHome.php?sid=17
} 
file with the date is obtained through an HTTP request issued by the Smart Grid Simulation Engine towards the school web site. The computation of the power produced by a wind turbine given the wind speed sensed is performed by the Energy Calculator Engine component. The component uses the wellknow relationship between wind speed and power extracted by a wind turbine: $P=\frac{1}{2} \rho A U^{3} C_{p}$ where $\rho$ is the air density, $A$ is the rotor swept area, $U$ is the wind speed and $C_{p}$ is the power coefficient representing the efficiency of the turbine rotor [29]. Once we have chosen the turbine, the parameters are known: $A=\pi\left(\frac{3.5}{2}\right)^{2}$ (the turbine blades have a 3.5 meters diameter), $\rho=1.225$ (typical air density value), $C_{p}=0.35$ (a typical value of rotor efficiency for wind turbines) and the wind speeds (i.e., cut-in and cut-out speed) between which the turbine works, then the wind to power relationship can be applied. Also in this case we assume to have the data about the wind speed available every hour and constant.

\subsection{Pricing energy from renewables}

Usually renewable sources are considered as base load in energy dispatching mechanisms: when energy is generated by these types of plants it is fed into the system and these plants are never shut down [30]. In our Smart Home, we consider both solar and wind power to have a cost of production due to the investment that is required to purchase, install and maintain the equipment.

The idea, and the related computations performed by the Price Extractor Engine consider a simplified investment analysis for calculating the cost for each kWh of produced energy. We simply consider the investment cost, the maintenance and operations costs, governmental incentives and the energy produced over the expected lifetime of the plant. Therefore we have the energy cost coming from the PV as $E C_{P V}=\left(C_{I n v}+C_{O p r}+C_{M a i}\right) / E n_{L}$ where $C_{I n v}$ is the total investment cost for the PV array, $C_{O p r}$ and $C_{M a i}$ are the total cost of operation and the total maintenance cost respectively over the investment lifetime for the PV system. For the PV array we consider 20 years of service for the AP-100 model. $E n_{L}$ is the estimated overall energy to be produced during the lifetime of the PV array. First, we estimate a production of energy during the 20 years of panel lifetime that is on average the same as the one produced in the previous years since the installation at Dalton school. Second, the investment cost is based on the results by Wise et al. [31] that investigated the cost of PV panels in the U.S.A. The value that emerges from their analysis considering the cost for PV panels, inverters and installation once the incentives applied by the U.S. government are subtracted, is 5.1 dollar for each installed watt of power. Third, the maintenance and operation costs can be considered as an annual expense about $0.84 \%$ of the investment, as found by Rehman et al. [32] for a $\mathrm{PV}$ installation. Another component that might be associated to operation and maintenance costs is the replacement of inverters which, as suggested in Croxford et al. [33], should be considered every 10 years. According to the findings of Wiser et al. [31] inverters replacement amounts about $7 \%$ of the initial total investment in a PV project for residential and small commercial purposes. Once we feed these parameters in the Energy Calculator Engine component it provides a constant value that represents the cost of each $\mathrm{kWh}$ generated by the PV plant. Therefore, the PV plant can be considered as an additional "virtual" energy provider since the produced energy in a future deregulated Smart Grid 
could be sold on the market at the price of returning from the investment (i.e., obtained from the computation shown above).

We apply a similar relationship to evaluate the cost of electricity generated by the small-wind turbine: $E C_{S W}=\left(C_{I n v}+C_{O p r}+C_{M a i}-I_{G o v}\right) / E n_{L}$. The only parameter that we have in addition to those described above is $I_{\text {Gov }}$ that represents the incentive subsidy from the government in wind energy. We consider an investment cost for the wind turbine of $28000 \$$ comprising the installation and required equipment (e.g., installation pole, inverters, workforce) and an annual cost of maintenance and operations of $1 \%$ of the total investment. This is in line with the findings of [34]. $E n_{L}$ for the turbine is the estimated overall energy to be produced during its lifetime (according to [35] the lifetime of a wind turbine can be considered around 20 years). To estimate the production of energy in New York we consider the average wind speed recorded by NOAA in more than 50 years. ${ }^{11}$ Using this data the turbine produces around 1300 $\mathrm{kWh}$ of energy per year. Considering the subsidy, the state of New York has an incentive program for small-scale wind installation to promote such energy source with a contribution for one year of $3.50 \$$ per kWh produced. ${ }^{12}$ Therefore, with all this parameters fed in the Energy Calculator Engine component one has once again a constant price for each $\mathrm{kWh}$ generated, in this case by the small-wind turbine.

\subsection{Renewable energy forecast}

The amount of energy that can be produced by renewable systems is strongly correlated to the meteorological conditions. It is beyond the scope of this paper to build an exact model of how meteorological and environmental conditions influence the energy production of wind and solar plants; here we aim at a realistic estimation of possible energy production in the broader context of simulation of realistic services for the Smart Grid. The Smart Grid Simulation Engine interacts with the NOAA national weather service ${ }^{13}$ web service requesting information about the wind speed, cloud coverage and temperatures. The service provides a week forecast given the location of interest (through longitude and latitude coordinates); for the first three days the forecast data has a 3 hours interval, while for the following days the interval is 6 hours. The Energy Forecast Engine has the task to adjust the meteorological data to an hour-by-hour information (in our simple solution we use interpolation) and to compute an hour-by-hour energy forecast for the week. The available power for each hour is computed in the following way:

- for the wind turbine, we apply $P=\frac{1}{2} \rho A U^{3} C_{p}$, given the wind speed from the forecast and the other wind turbine parameters;

- for solar panels, we apply $P=H_{p w 21}\left(C_{c}+T_{f}\right)$ where $H_{p w 21}$ is the hour-byhour average of the historical power produced the previous year during the 10 previous and 10 following days of the very same day of the previous year. Therefore, we have an average value of produced power in comparable days, i.e., similar sun horizon condition. The terms in brackets $C_{c}$ and $T_{f}$

\footnotetext{
${ }^{11}$ http://1wf.ncdc.noaa.gov/oa/climate/online/ccd/avgwind.html

${ }^{12} \mathrm{http}: / /$ ww. dsireusa.org/incentives/incentive. $\mathrm{cfm}$ ? Incentive_Code=NY35F\&re= O\&ee $=0$

13 http://graphical. weather.gov/xml/
} 
are used to correct the power considering cloud coverage and temperature estimations. $C_{c}$ represents the percentage of cloud coverage and $T_{f}=$ $\left(T_{\text {his }}-T\right) \delta$ is a temperature factor that considers, based on the difference in the average hourly temperature in the same 21-day period in the previous year, the increase/decrease in efficiency of $\delta$ percentage. We consider a linear relation which establishes a decrease/increase of $\delta=0.5 \% /{ }^{\circ} \mathrm{C}$ in PV efficiency for a polycrystalline silicon panel as the temperature increases/decreases compared to factory test conditions [36].

Following these models the Energy Forecast Engine provides for one week available power hour-by-hour for the two sources of renewable energy installed in our hypothetical Smart Home.

\section{Results}

The system and its components as described in Sections 3 and 4 have been extensively used in a realization of a Smart Office at the University of Groningen. The goal of the Smart Office is to minimize the energy bill for electricity without compromising the well-being and productivity of the workers. For the detailed results of the experiment, which are beyond the scope of the present paper, we refer to [7]. Here we give a glimpse of the savings achieved in terms of money and energy in Table 2. The table shows the savings when the operation of a set of office appliances such as the laptop, printer, microwave, are mandated by a computer application that schedules their operations. Such scheduler has the objective of minimizing the expenses in the usage of the appliances without compromising well-being and productivity of the users. The savings come from taking advantage of the lower prices in the Dynamic Pricing range obtained from the Price Extractor Engine (cf. Section 4). The first line of Table 2 considers the possibility of using locally produced energy through renewables, the data of this own produced energy is obtained through the Energy Calculator Engine and the prices of solar and wind energy as proposed in Section 4. The second line is a worst scenario where no locally produced energy is available. Here we do not stress the benefits achieved (economic and energetic), but the focus is that the realistic services of the future Smart Grid implemented can be used to test appliances, optimizers, schedulers and Smart Grid scenarios based on real data.

\begin{tabular}{|l|l|l|}
\hline & $\begin{array}{l}\text { Avg. Mone- } \\
\text { tary savings }\end{array}$ & $\begin{array}{l}\text { Avg. Energy } \\
\text { savings }\end{array}$ \\
\hline $\begin{array}{l}\text { Dynamic Pricing and } \\
\text { local renewables }\end{array}$ & $35 \%$ & $10 \%$ \\
\hline $\begin{array}{l}\text { Dynamic Pricing and } \\
\text { without local renew- } \\
\text { ables }\end{array}$ & $20 \%$ & $10 \%$ \\
\hline
\end{tabular}

Table 2: Energy and monetary savings with dynamic pricing [7].

Figure 4 shows an example of the output of the Price Extractor component of the Smart Grid Emulation Engine. It represents the price of electricity on the PJM market for a sub set of the zones in the U.S. electrical system. One notes 
that there are significant variations in price during the day: from the minimum in the middle of the night, the price almost doubles in the evening. Therefore, with such information available beforehand (we recall that these prices are dayahead) one can create automation to provide consistent savings over time. Think for instance of the scheduling of a washing machine, a dryer and a dishwasher or the charging of an electric car.

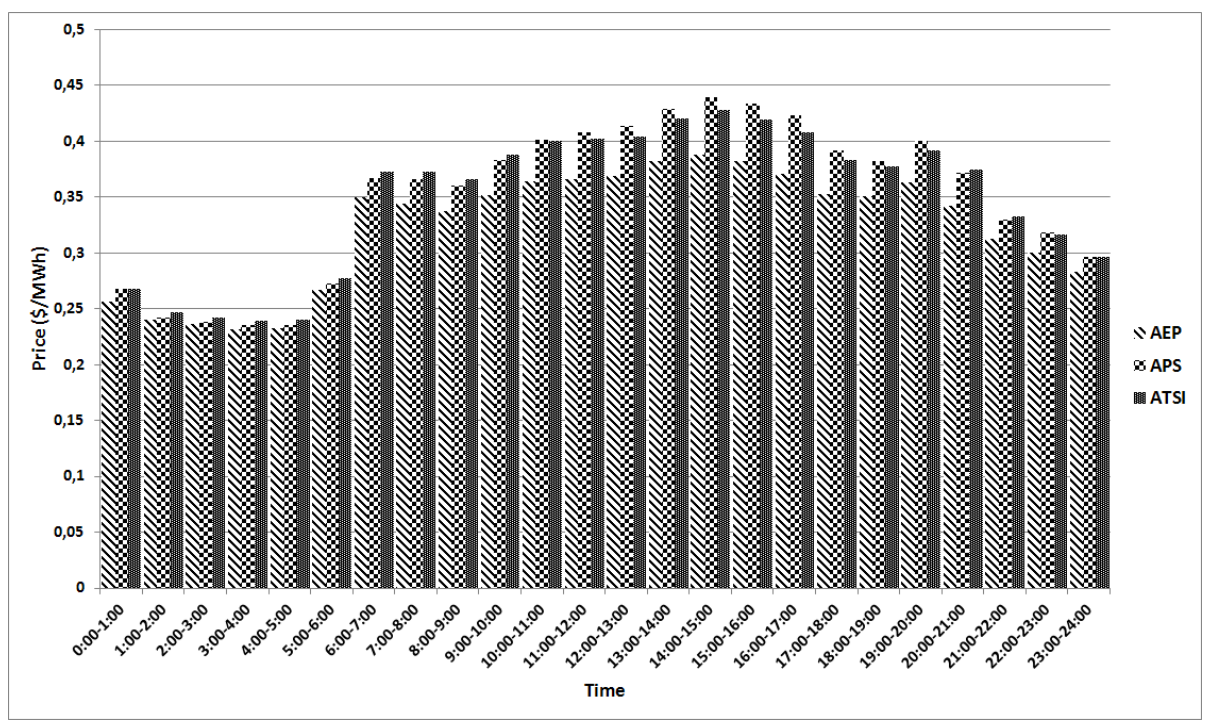

Figure 4: Energy prices for several locations of the U.S. electrical system on $5^{\text {th }}$ October 2012.

Having information about the amount of renewable energy to be produced (forecast) is essential to better plan on a week basis the activities that can wait the optimal environmental conditions for self production. Figure 5 shows the amount of energy that is forecast at the installation site we consider (Dalton school) and by using the weather forecast of NOAA and the model proposed in Section 4. In the figure, one week of power forecast is shown. The continuous line represents the power provided by the PV installation with a typical bellshaped power output during the daytime and flat night profile. The dashed line represents the forecast of the wind turbine that has an irregular pattern. One can see how $6^{\text {th }}, 7^{\text {th }}$ and $10^{\text {th }}$ October are more favorable to the self-production of energy by using solar panels, while the night between $6^{\text {th }}$ and $7^{\text {th }}$ and the days $10^{t h}$ and $11^{t h}$ are very favorable to power production through the wind turbine. By exploiting this information the Smart Home can have an idea on when to schedule the usage of devices.

Naturally, in an implementation of the Smart Grid services on the field, a reconciliation system to evaluate the gaps between forecast and real prices will be needed a posteriori for billing purposes and to inform the user. The same reconciliation will be required in the actual amount of energy extracted from the Grid in case of non exact forecasts of generated renewable energy. This aspect is out of the scope of the present paper, however we envision that the Smart Meter will be the center of this reconciliation by storing the in- and out-flow of energy and the tariffs applied at each point in time. 


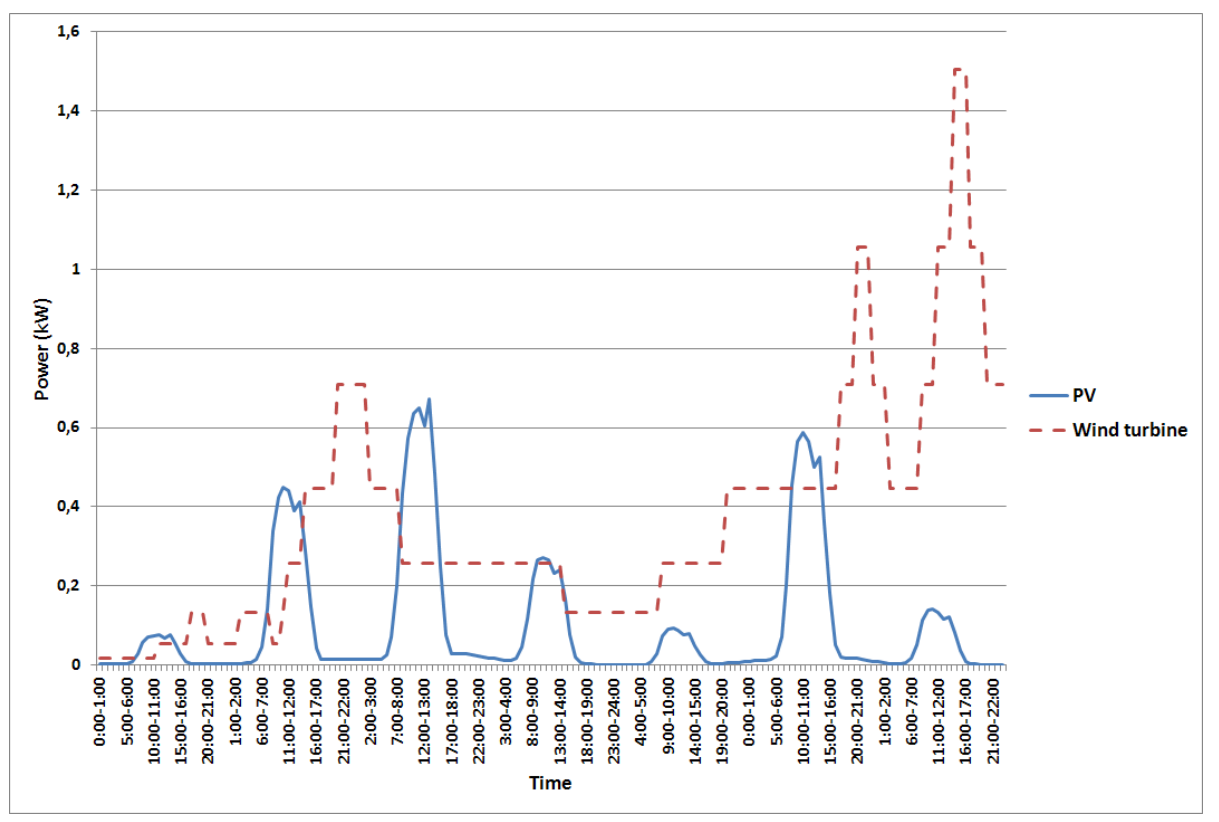

Figure 5: Energy forecast for solar power and wind power in the week $5^{t h}-11^{t h}$ October 2012.

\section{Concluding Remarks}

The Smart Grid as much as it is envisioned and researched is though not yet a reality. In fact, this system promises to be one of the most complex in terms of size, actors involved and number of independent subsystems, though it has not yet been investigated as such. To be able to progress in the research and development, accurate simulation tools are necessary. In the present work, we have designed and implemented a simulation component that, taking data from live sources about energy prices, environmental conditions, and renewable generation, can simulate Dynamic Pricing of energy from multiple vendors. Such solution is interesting for testing purposes in pilot projects, or to evaluate user experience in a dynamic tariff environment.

We note that the energy prices come from wholesale energy markets, these prices are indeed a good indication of price evolutions, but unfortunately they are not the prices reaching the end-user which are just a reflection of those. More aspects could be taken into account to make these prices be closer to the actual end-user price, such as distance from the source of the selling party, or dispatching and distribution costs. With more advances in Smart Grid standardization and with more real Smart Grid implementations on the field, we envision a growth in Smart Grid service providers that will give information in an easy accessible fashion.

The effort of the present work goes in the direction of anticipating the services required by the new ICT-related subsystems of the future Smart Grid to enable the user's direct involvement in the Demand-Response mechanism. Based on real information coming from existing on-line services, we have simulated the main sets of functionality to implement Demand-Response: Dynamic 
Pricing, energy self production and energy production forecast. The simple system realized has been successfully used in a real Demand-Response experiment to evaluate potential savings of Dynamic Pricing [7]. We believe though that the system can be more globally beneficial for Smart Grid testing.

\section{Acknowledgment}

The work is supported by the EU FP7 Project GreenerBuildings, contract no. 258888 and by the Dutch National Research Council under the NWO Smart Energy Systems programme, contract no. 647.000.004. Pagani is supported by University of Groningen with the Ubbo Emmius Fellowship 2009.

\section{References}

[1] M. G. Morgan, J. Apt, L. B. Lave, M. D. Ilic, M. Sirbu, and J. M. Peha, "The many meanings of "smart grid"," Carnegie Mellon University, Tech. Rep., 2009.

[2] National Energy Technology Laboratory, "A system view of the modern grid," U.S. Department of Energy - Office of Electricity Delivery and Energy Reliability, Tech. Rep., 2007.

[3] _ , "A vision for the modern grid," U.S. Department of Energy - Office of Electricity Delivery and Energy Reliability, Tech. Rep., 2007.

[4] A.-H. Mohsenian-Rad, V. Wong, J. Jatskevich, and R. Schober, "Optimal and autonomous incentive-based energy consumption scheduling algorithm for smart grid," in Innovative Smart Grid Technologies (ISGT), 2010, jan. 2010, pp. $1-6$.

[5] International Eenrgy Agency, "World energy outlook," International Eenrgy Agency, Tech. Rep., 2009.

[6] M. Hand, S. Baldwin, E. DeMeo, J. Reilly, T. Mai, D. Arent, G. Porro, M. Meshek, and D. Sandor, "Renewable electricity futures study," NREL Paper, 2012, national Renewable Energy Laboratory.

[7] I. Georgievski, V. Degeler, G. A. Pagani, T. A. Nguyen, A. Lazovik, and M. Aiello, "Optimizing energy costs for offices connected to the smart grid," Smart Grid, IEEE Transactions on, To Appear/DOI:10.1109/TSG.2012.2218666 2012.

[8] A. Schuelke and K. Erickson, "Serving solar variations with consumption control of smart appliances and electric vehicles," in Innovative Smart Grid Technologies (ISGT Europe), 2011 2nd IEEE PES International Conference and Exhibition on, dec. 2011, pp. 1 -8.

[9] D. Wang, B. de Wit, S. Parkinson, J. Fuller, D. Chassin, C. Crawford, and N. Djilali, "A test bed for self-regulating distribution systems: Modeling integrated renewable energy and demand response in the gridlab-d/matlab environment," in Innovative Smart Grid Technologies (ISGT), 2012 IEEE PES, jan. 2012, pp. $1-7$. 
[10] S. Karnouskos, "Future smart grid prosumer services," in Innovative Smart Grid Technologies (ISGT Europe), 2011 2nd IEEE PES International Conference and Exhibition on, dec. 2011, pp. 1 -2.

[11] S. Karnouskos, P. Da Silva, and D. Ilic, "Energy services for the smart grid city," in Digital Ecosystems Technologies (DEST), 2012 6th IEEE International Conference on, june 2012, pp. 1 -6.

[12] T. Verschueren, W. Haerick, K. Mets, C. Develder, F. De Turck, and T. Pollet, "Architectures for smart end-user services in the power grid," in Network Operations and Management Symposium Workshops (NOMS Wksps), 2010 IEEE/IFIP, april 2010, pp. $316-322$.

[13] N. Capodieci, E. Alsina, and G. Cabri, "A context-aware agent-based approach for deregulated energy market," in Enabling Technologies: Infrastructure for Collaborative Enterprises (WETICE), 2012 IEEE 21st International Workshop on, june 2012, pp. $16-21$.

[14] J. Byun, I. Hong, B. Kang, and S. Park, "A smart energy distribution and management system for renewable energy distribution and contextaware services based on user patterns and load forecasting," Consumer Electronics, IEEE Transactions on, vol. 57, no. 2, pp. 436 -444, may 2011.

[15] C. Roe, S. Meliopoulos, R. Entriken, and S. Chhaya, "Simulated demand response of a residential energy management system," in Energytech, 2011 IEEE, may 2011, pp. $1-6$.

[16] N. Venkatesan, J. Solanki, and S. Solanki, "Market optimization for microgrid with demand response model," in North American Power Symposium (NAPS), 2011, aug. 2011, pp. $1-6$.

[17] A. Khan and H. Mouftah, "Web services for indoor energy management in a smart grid environment," in Personal Indoor and Mobile Radio Communications (PIMRC), 2011 IEEE 22nd International Symposium on, sept. 2011, pp. $1036-1040$.

[18] O. Asad, M. Erol-Kantarci, and H. Mouftah, "Sensor network web services for demand-side energy management applications in the smart grid," in Consumer Communications and Networking Conference (CCNC), 2011 IEEE, jan. 2011, pp. $1176-1180$.

[19] G. A. Pagani and M. Aiello, "Service orientation and the smart grid state and trends," Service Oriented Computing and Applications, vol. 6, no. 3, pp. 267-282, 2012.

[20] S. Sucic, A. Martinic, and A. Kekelj, "Utilizing standards-based semantic services for modeling novel smart grid supervision and remote control frameworks," in Industrial Technology (ICIT), 2012 IEEE International Conference on, march 2012, pp. $409-414$.

[21] G. A. Pagani and M. Aiello, "Towards a service-oriented energy market: Current state and trend," in Service-Oriented Computing, ser. Lecture Notes in Computer Science, E. Maximilien, G. Rossi, S.-T. Yuan, H. Ludwig, and M. Fantinato, Eds. Springer Berlin / Heidelberg, 2011, vol. 6568, pp. 203-209. 
[22] S. Karnouskos, A. Weidlich, K. Kok, C. Warmer, J. Ringelstein, P. Selzam, A. Dimeas, and S. Drenkard, "Field trials towards integrating smart houses with the smart grid," in Energy-Efficient Computing and Networking, ser. LNCS. Springer, 2011, vol. 54, pp. 114-123.

[23] F. Bouffard, "The challenge with building a business case for smart grids," in Power and Energy Society General Meeting, 2010 IEEE, july 2010, pp. $1-3$.

[24] National Energy Technology Laboratory, "Building a smart grid business case," U.S. Department of Energy - Office of Electricity Delivery and Energy Reliability, Tech. Rep., 2009.

[25] M. Papazoglou, Web Services: Principles and Technology. Pearson, 2007.

[26] G. Lu, D. De, and W.-Z. Song, "Smartgridlab: A laboratory-based smart grid testbed," in Smart Grid Communications (SmartGridComm), 2010 First IEEE International Conference on, oct. 2010, pp. 143 -148.

[27] W. Taqqali and N. Abdulaziz, "Smart grid and demand response technology," in Energy Conference and Exhibition (EnergyCon), 2010 IEEE International, dec. 2010, pp. $710-715$.

[28] K. Kok, Z. Derzsi, M. Hommelberg, C. Warmer, R. Kamphuis, and H. Akkermans, "Agent-based electricity balancing with distributed energy resources, a multiperspective case study," in Hawaii International Conference on System Sciences, Proceedings of the 41st Annual, jan. 2008, p. 173.

[29] L. Freris and D. Infield, Renewable Energy in Power Systems. W, 2008.

[30] D. T. Swift-Hook, "Grid-connected intermittent renewables are the last to be stored," Renewable Energy, vol. 35, no. 9, pp. 1967 - 1969, 2010.

[31] R. Wiser, G. Barbose, and C. Peterman, "Tracking the sun: the installed cost of photovoltaics in the u.s. from 1998-2007," Lawrence Berkeley National Laboratory, Tech. Rep., 2009.

[32] S. Rehman, M. A. Bader, and S. A. Al-Moallem, "Cost of solar energy generated using pv panels," Renewable and Sustainable Energy Reviews, vol. 11 , no. 8 , pp. $1843-1857,2007$.

[33] B. Croxford and K. Scott, "Can pv or solar thermal systems be cost effective ways of reducing co 2 emissions for residential buildings?" in Solar 2006: Renewable Energy - Key to Climate Recovery, ser. American Solar Energy Society, 2006.

[34] C. A. Walford, "Wind turbine reliability: understanding and minimizing wind turbine operation and maintenance costs," Sandia National Laboratries, Tech. Rep. SAND2006-1100, 2006.

[35] "Life cycle assessment of a wind farm and related externalities," Renewable Energy, vol. 20, no. 3, pp. 279 - 288, 2000. 
[36] D. King, W. Boyson, and J. Kratochvil, "Analysis of factors influencing the annual energy production of photovoltaic systems," in Photovoltaic Specialists Conference, 2002. Conference Record of the Twenty-Ninth IEEE, may 2002, pp. $1356-1361$. 